\title{
LETTER \\ Design of a Multiple-Input SC DC-DC Converter Realizing Long Battery Runtime
}

\author{
Kei EGUCHI $^{\dagger a)}$, Member, Sawai PONGSWATD ${ }^{\dagger \dagger}$, Amphawan JULSEREEWONG ${ }^{\dagger \dagger}$, \\ Kitti TIRASESTH $^{\dagger \dagger}$, Nonmembers, Hirofumi SASAKI ${ }^{\dagger \dagger}$, and Takahiro INOUE ${ }^{\dagger \dagger \dagger}$, Members
}

\begin{abstract}
SUMMARY A multiple-input switched-capacitor DC-DC converter which can realize long battery runtime is proposed in this letter. Unlike conventional converters for a back-lighting application, the proposed converter drives some LEDs by converting energy from solar cells. Furthermore, the proposed converter can charge a lithium battery when an output load is light. The validity of circuit design is confirmed by theoretical analyses, simulations, and experiments.

key words: DC-DC converters, switched-capacitor circuits, back-lighting applications, clean energy
\end{abstract}

\section{Introduction}

A switched-capacitor (SC) DC-DC converter [1]-[10] has been used as a driver circuit of white LEDs for display back-lighting. Because the SC converter can realize thin circuit composition, light-weight, no flux of magnetic induction, and so on. In mobile back-lighting applications, the stepped-up voltage such as $4.75-6.5 \mathrm{~V}$ (Typ. $=5 \mathrm{~V}$ ) is required to drive LEDs at up to $25 \mathrm{~mA}$. For this reason, SC converters realizing $2 \times$ or $1.5 \times$ step-up conversion [5]-[8] have been used to drive LEDs. However, it is difficult to improve power efficiency further by adjusting the ratio of the voltage conversion, because the conversion ratio is predetermined by circuit structure of the SC power converter.

To solve this problem, a multiple-input SC DC-DC converter realizing long battery-runtime is proposed in this letter. Unlike conventional SC power converters [1]-[10], the proposed converter drives LEDs by converting energy from solar cells when solar cells offer enough energy. Furthermore, the proposed converter can charge a lithium battery when an output load is light. Hence, the proposed converter enables us to realize long battery runtime. To confirm the validity of the circuit design, theoretical analyses, SPICE simulations, and experiments are performed.

\footnotetext{
Manuscript received September 25, 2009.

Manuscript revised January 17, 2010.

${ }^{\dagger}$ The author is with the Department of Technology Education, Shizuoka University, Shizuoka-shi, 422-8529 Japan.

${ }^{\dagger \dagger}$ The authors are with the Faculty of Engineering, King Mongkut's Institute of Technology Ladkrabang, Ladkrabang, Bangkok, 10525, Thailand.

$t^{\dagger \dagger}$ The author is with the School of Industrial Engineering, Tokai University, Kumamoto-shi, 862-8652 Japan.

${ }^{+1+\dagger}$ The author is with the Department of Electrical and Computer Engineering, Kumamoto University, Kumamoto-shi, 8608555 Japan.

a) E-mail: ekeguch@ipc.shizuoka.ac.jp

DOI: 10.1587/transfun.E93.A.985
}

\section{Circuit Structure}

\subsection{Conventional Converter}

Figure 1 shows an example of conventional converters for mobile back-lighting applications. The converter shown in Fig. 1 is one of the most popular converters and is adopted in MAX1910, MAX1912*, and so on. As shown in Fig. 1, power switches $S_{1}-S_{7}$ are driven by non-overlapped 2phase clock pulses synchronously. Since the conversion ratio is predetermined by the circuit structure, the conventional converter is difficult to improve power efficiency by adjusting the conversion ratio.

\subsection{Proposed Converter}

Figure 2 shows a general form of the proposed converter. The proposed converter consists of $5 N+7(N=1,2, \ldots)$ power switches and $N+2$ capacitors. As Fig. 2 shows, the proposed converter has two kinds of different inputs: $V_{i n 1}$ which is offered by a lithium battery and $V_{i n 2}$ which is supplied by solar cells. When solar cells offer enough electric power, the proposed converter drives LEDs by converting solar energy input $V_{i n 2}$. Furthermore, the lithium battery is charged when the output load is light. Only when the solar cells cannot generate enough electric power, the LEDs

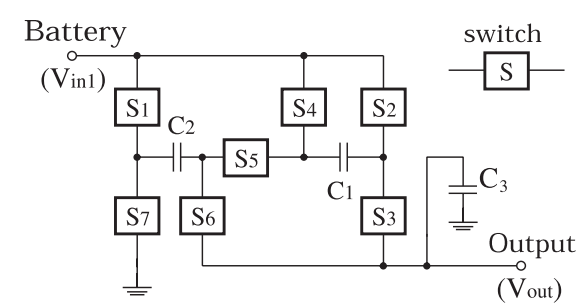

\begin{tabular}{|c|c|c|c|}
\hline Mode & Phase & On & Off \\
\hline \hline \multirow{2}{*}{$2 \times$} & Charging & $S_{3}, S_{4}, S_{5}, S_{7}$ & Other switches \\
\cline { 2 - 4 } & Transfer & $S_{1}, S_{3}, S_{4}, S_{6}$ & Other switches \\
\hline \multirow{2}{*}{$1.5 \times$} & Charging & $S_{2}, S_{5}, S_{7}$ & Other switches \\
\cline { 2 - 4 } & Transfer & $S_{1}, S_{3}, S_{4}, S_{6}$ & Other switches \\
\hline
\end{tabular}

Fig. 1 Conventional DC-DC converter realizing $1.5 \times / 2 \times$ step-up conversion.

${ }^{*}$ MAX1910 and MAX1912 are $1.5 \times / 2 \times$ step-up converters produced by Maxim Integrated Products. 


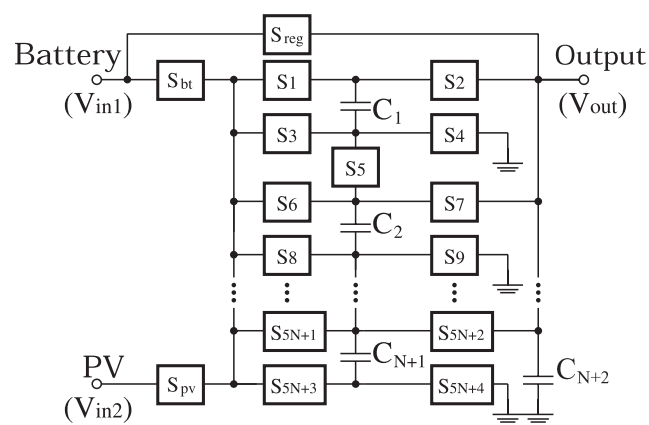

Fig. 2 General form of proposed SC DC-DC converter.

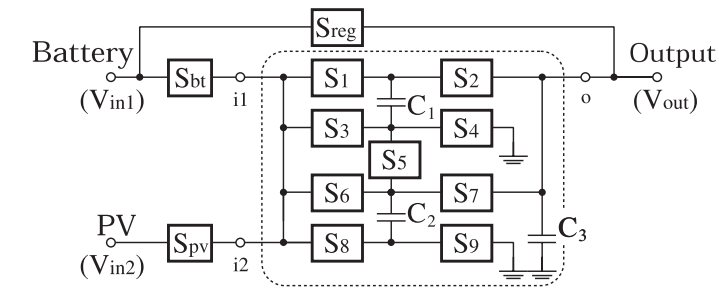

\begin{tabular}{|c|c|c|c|}
\hline Input & Mode & Phase & On* \\
\hline \multirow{2}{*}{$V_{\text {in } 2}$} & \multirow{2}{*}{$3 \times$} & Charging & 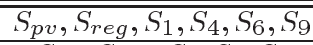 \\
\hline & & Transfer & $S_{p v}, S_{r \in g}, S_{2}, S_{5}, S_{8}$ \\
\hline \multirow{2}{*}{$V_{\text {in } 2}$} & \multirow{2}{*}{$2 \times$} & Charging & $S_{p v}, S_{r \in g}, S_{1}, S_{4}, S_{7}, S_{8}$ \\
\hline & & Transfer & $S_{p v}, S_{r e g}, S_{2}, S_{3}, S_{6}, S_{9}$ \\
\hline \multirow{2}{*}{$V_{\text {in } 2}$} & \multirow{2}{*}{$1.5 \times$} & Charging & $S_{p v}, S_{r \in g}, S_{1}, S_{5}, S_{9}$ \\
\hline & & Transfer & $S_{p v}, S_{r \in g}, S_{2}, S_{3}, S_{7}, S_{8}$ \\
\hline \multirow{2}{*}{$V_{\text {in } 1}$} & \multirow{2}{*}{$1.5 \times$} & Charging & $S_{b t}, S_{1}, S_{5}, S_{9}$ \\
\hline & & Transfer & $S_{b t}, S_{2}, S_{3}, S_{7}, S_{8}$ \\
\hline
\end{tabular}

Fig. 3 Example of proposed converter when $N=1$.

are driven by converting the energy from the lithium battery. Hence, the proposed converter enables us to realize long battery runtime.

In the following section, to help readers' understanding, theoretical analyses will be performed concerning the simplest converter shown in Fig. 3.

\section{Theoretical Analysis}

According to $V_{i n 2}$, the proposed converter shown in Fig. 3 offers 4 kinds of conversion modes. When $V_{i n 1} / 2 \leq V_{\text {in } 2}<$ $3 V_{i n 1} / 4,3 V_{i n 1} / 4 \leq V_{i n 2}<V_{i n 1}$, and $V_{i n 1} \leq V_{i n 2}$, the proposed converter realizes $3 \times, 2 \times$, and $1.5 \times$ step-up conversion, respectively, where the input voltage is $V_{i n 2}$. On the other hand, the proposed converter realizes $1.5 \times$ step-up conversion when $V_{i n 2}<V_{i n 1} / 2$, where the input voltage is $V_{i n 1}$. Figure 4 shows instantaneous equivalent circuits when $3 \times$ step-up mode.

First, the equivalent circuit when $3 \times$ step-up conversion is analyzed. In the theoretical analysis, we assume that 1. parasitic elements are negligibly small and 2. time constant is much larger than the period of clock pulses. In the steady state of Fig. 4, differential values of electric charges in $C_{k}(k=1,2,3)$ satisfy

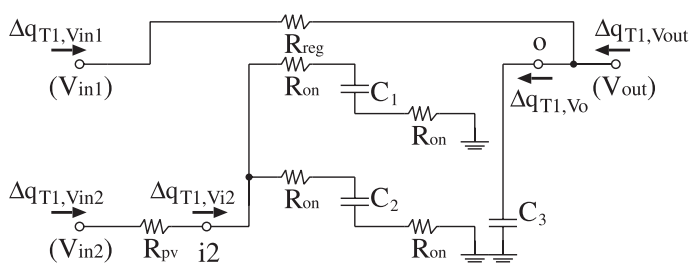

(a)

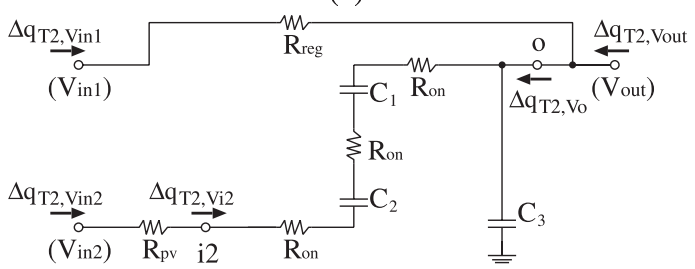

(b)

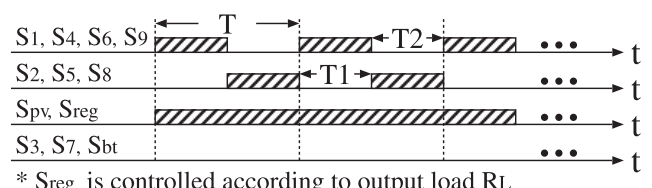

(c)

Fig. 4 Instantaneous equivalent circuits when $V_{i n 1} / 2 \leq V_{i n 2}<3 V_{i n 1} / 4$. (a) State $-T 1$. (b) State $-T 2$. (c) Timing of pulses.

$$
\Delta q_{T 1}^{k}+\Delta q_{T 2}^{k}=0
$$

where $\Delta q_{T 1}^{k}$ and $\Delta q_{T 2}^{k}$ denote electric charges when State $T 1$ and $S$ tate $-T 2$, respectively. In the case of $S$ tate $-T 1$, differential values of the electric charges in terminal- $i 2$ and terminal- $o, \Delta q_{T 1, V_{i 2}}$ and $\Delta q_{T 1, V_{o}}$, are given by

$$
\begin{aligned}
\Delta q_{T 1, V_{12}} & =\Delta q_{T 1}^{1}+\Delta q_{T 1}^{2} \\
\text { and } \Delta q_{T 1, V_{o}} & =\Delta q_{T 1}^{3} .
\end{aligned}
$$

On the other hand, in the case of $S$ tate $-T 2$, differential values of electric charges in terminal- $i 2$ and terminal- $o$ (see in Fig. 4), $\Delta q_{T 2, V_{i 2}}$ and $\Delta q_{T 2, V_{o}}$, are given by

$$
\begin{aligned}
\Delta q_{T 2, V_{i 2}} & =-\Delta q_{T 2}^{1}=-\Delta q_{T 2}^{2} \\
\text { and } \Delta q_{T 2, V_{o}} & =\Delta q_{T 2}^{1}+\Delta q_{T 2}^{3} .
\end{aligned}
$$

Here, averaged currents of terminal- $i 2$ and terminal-o are given by

$$
\begin{aligned}
\overline{I_{i 2}} & =\left(\Delta q_{T 1, V_{i 2}}+\Delta q_{T 2, V_{i 2}}\right) / T=\Delta q_{V_{i 2}} / T \\
\text { and } \overline{I_{o}} & =\left(\Delta q_{T 1, V_{o}}+\Delta q_{T 2, V_{o}}\right) / T=\Delta q_{V_{o}} / T,
\end{aligned}
$$

where $\Delta q_{V_{i 2}}$ and $\Delta q_{V_{o}}$ are electric charges in terminal-i2 and terminal-o, respectively. From Eqs. (1)-(4), the following equation is derived:

$\overline{I_{i 2}}=-3 \overline{I_{o}}$.

In Fig. 3, the energy consumed by resistors in one period, $W_{T}$, can be expressed by

$$
W_{T}=W_{T 1}+W_{T 2},
$$

where $W_{T 1}=\frac{2 R_{o n}}{T 1}\left(\Delta q_{T 1}^{1}\right)^{2}+\frac{2 R_{o n}}{T 1}\left(\Delta q_{T 1}^{2}\right)^{2}$ 
and $\quad W_{T 2}=\frac{3 R_{o n}}{T 2}\left(\Delta q_{T 2}^{1}\right)^{2}$.

Here, it is known that a general equivalent circuit of SC power converters can be expressed by the determinant using a Kettenmatrix. In the general equivalent circuit [1], the consumed energy $W_{T}$ is defined by

$$
W_{T}=W_{T 1}+W_{T 2} \equiv\left(\frac{\Delta q_{V_{o}}}{T}\right)^{2} \cdot R_{S C} \cdot T,
$$

where $R_{S C}$ is called the SC resistance. By substituting Eqs. (2), (3), and (6) into (7), the SC resistance when the $3 \times$ step-up mode, $R_{S C, 3 \times}$, is obtained by

$$
R_{S C, 3 \times}=\frac{4-D}{D(1-D)} \cdot R_{o n},
$$

where $D(=T 1 / T)$ denotes the duty factor. Therefore, by using Eqs. (5) and (8), the equivalent circuit concerning the $3 \times$ conversion mode can be expressed by the circuit shown in Fig. 5. In Fig. 5, power efficiency $\eta_{3 \times}{ }^{\dagger}$ is obtained by $\eta_{3 \times}=P_{\text {out }} / P_{\text {total }}$, where

$$
\begin{aligned}
P_{\text {out }}= & R_{L}\left(\frac{R_{\text {reg }}}{R_{L}+R_{\text {reg }}}\right)^{2}\left(\overline{I_{o}}\right)^{2} \\
\text { and } P_{\text {total }}= & P_{\text {out }}+9 R_{p v}\left(\overline{I_{o}}\right)^{2}+R_{S C, 3 \times\left(\overline{I_{o}}\right)^{2}} \\
& +R_{\text {reg }}\left(\frac{R_{L}}{R_{L}+R_{\text {reg }}}\right)^{2}\left(\overline{I_{o}}\right)^{2} .
\end{aligned}
$$

Especially, power efficiency $\eta_{3 \times}$ can be expressed by

$$
\eta_{3 \times}=\frac{R_{L}}{R_{L}+9 R_{p v}+R_{S C, 3 \times}},
$$

when $R_{\text {reg }} \rightarrow \infty$.

To save space, only the characteristic of the $3 \times$ step-up mode is discussed in this manuscript. However, the characteristics of other conversion modes can also be analyzed by the same method. Table 1 shows the summary of the theoretical results concerning other conversion modes of the

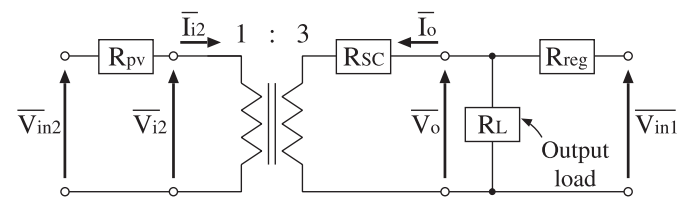

Fig. 5 Equivalent circuit of proposed converter when $3 \times$ step-up mode.

Table 1 Summary of theoretical results.

\begin{tabular}{|c|c|c|}
\hline Input range & Ratio & $R_{S C}$ \\
\hline \hline$V_{i n 1} / 2 \leq V_{i n 2}<3 V_{i n 1} / 4$ & $3 \times$ & $\frac{4-D}{D(1-D)} \cdot R_{o n}$ \\
\hline $3 V_{i n 1} / 4 \leq V_{i n 2}<V_{i n 1}$ & $2 \times$ & $\frac{1}{D(1-D)} \cdot R_{o n}$ \\
\hline$V_{i n 1} \leq V_{i n 2}$ & $1.5 \times$ & $\frac{3+D}{4 D(1-D)} \cdot R_{o n}$ \\
\hline$V_{i n 1} / 2>V_{i n 2}$ & $1.5 \times$ & $\frac{3+D}{4 D(1-D)} \cdot R_{o n}$ \\
\hline
\end{tabular}

proposed converter.

\section{Simulation \& Experiment}

To verify the theoretical analyses, SPICE simulations were performed under conditions where $N=1, C_{1}-C_{3}=2 \mu \mathrm{F}$, $D=0.5, T=1 \mu \mathrm{s}$ and $R_{o n}=0.8 \Omega$. Figure 6 shows the power efficiency of the proposed converter as a function of output load $R_{L}$, where $R_{r e g}$ was set to $R_{r e g}=\infty$. As Fig. 7 shows, theoretical results agree well with simulated results. To evaluate the power efficiency, the theoretical analysis was performed concerning conventional converter MAX1910 shown in Fig. 1. Table 2 shows the theoretical results of the conventional converter. As Tables 1 and 2 show, the power efficiency of the proposed converter is almost the same as that of the conventional converter, because the $\mathrm{SC}$ resistances are almost the same. However, unlike the conventional converter, the proposed converter can provide long battery runtime by converting clean energy input.

Next, to confirm the validity of circuit design, experiments were performed regarding to the proposed converter with $N=1$. The experimental circuit was built with commercially available transistors on a bread board. Figure 7 shows the $3 \times$ stepped-up voltage obtained by the experimental circuit, where $V_{i n 1}=0 \mathrm{~V}, V_{i n 2}=4 \mathrm{~V}, C_{1}-C_{3}=2.2 \mu \mathrm{F}$, $D=0.5, T=1 \mathrm{~ms}$ and $R_{L}=10 \mathrm{k} \Omega$. To save space, only the $3 \times$ stepped-up voltage is shown in this manuscript. Fig-

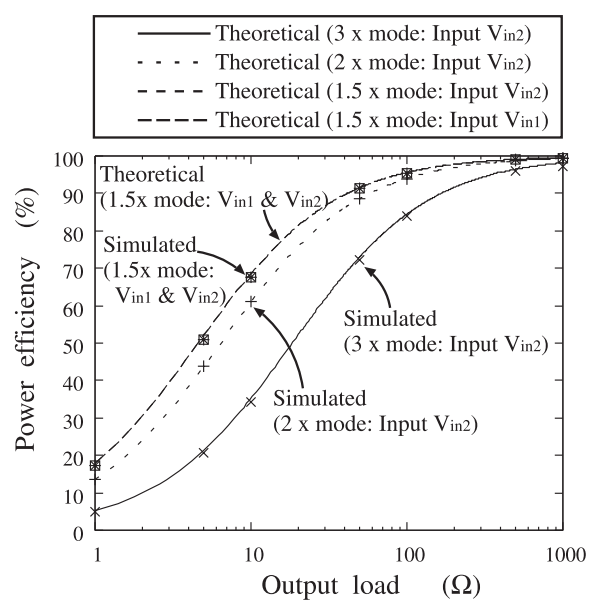

Fig. 6 Power efficiency of proposed converter.

Table 2 Theoretical results of conventional converter.

\begin{tabular}{|c|c|}
\hline Ratio & $R_{S C}$ \\
\hline \hline $2 \times$ & $\frac{3-D}{D(1-D)} \cdot R_{o n}$ \\
\hline $1.5 \times$ & $\frac{3+D}{4 D(1-D)} \cdot R_{o n}$ \\
\hline
\end{tabular}

'Of course, the consumed energy of peripheral circuits such as pulse generators, comparators, etc. is disregarded in the power efficiency. 


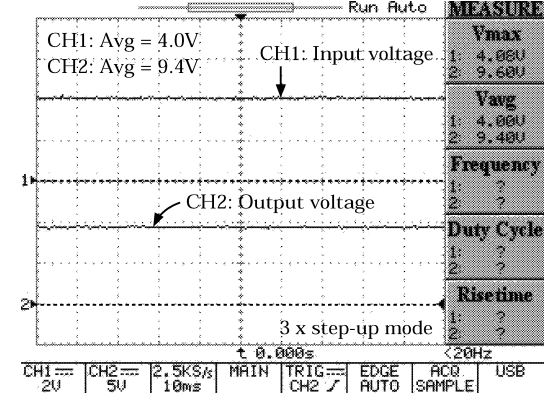

Fig. $73 \times$ step-up mode obtained by experimental circuit.

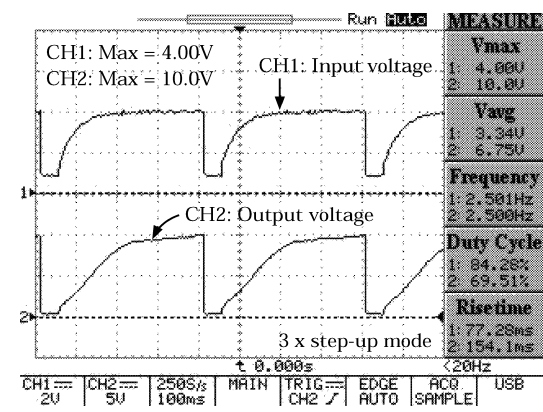

Fig. 8 Charge process obtained by experiment.

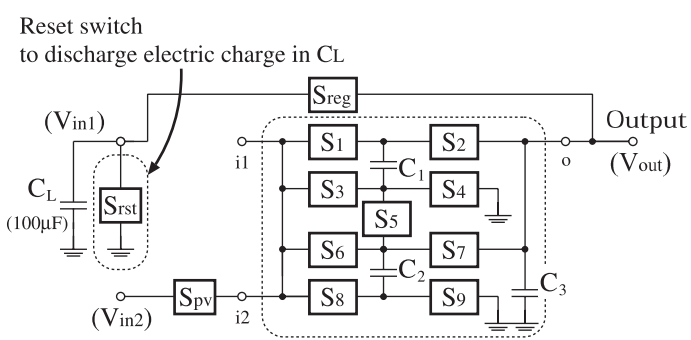

Fig. 9 Experimental circuit to investigate charge process.

ure 8 shows the charge process obtained by the experiment ${ }^{\dagger}$, where capacitor $C_{L}=100 \mu \mathrm{F}$ was connected to terminal$V_{i n 1}$ in substitution for the lithium battery to reduce the experiment time. In the experiment of Fig. 8, to confirm the charge process, reset switch $S_{r s t}$ was attached to terminal$V_{i n 1}$ as shown in Fig. 9. As Fig. 8 shows, the proposed con-

${ }^{\dagger}$ When $R_{L}=10 \mathrm{k} \Omega$, the power efficiency of the experimental circuit was $48.6 \%$, where input power $P_{\text {in }}$ was $3.7 \mathrm{~mW}$ and output power $P_{\text {out }}$ was $1.8 \mathrm{~mW}$. However, the power efficiency will be improved by integrating the proposed converter into an IC chip. Because, unlike an IC chip, the parasitic resistance of the bread board is very large. Therefore, only the circuit design was verified in this experiment. verter can charge the battery when the output load is light. Therefore, a long battery runtime will be realized by the proposed converter.

\section{Conclusion}

For the back-lighting application, a multiple-input switchedcapacitor (SC) DC-DC converter realizing long batteryruntime has been proposed in this paper.

Concerning power efficiency, derived theoretical formulas will be helpful to estimate circuit characteristics, because theoretical results agreed well with SPICE simulation results. Furthermore, the proposed converter enables us to realize long battery runtime, because the battery charge process was confirmed through experiments.

\section{References}

[1] N. Hara, I. Oota, I. Harada, and F. Ueno, "Programmable ring type switched-capacitor DC-DC converters," IEICE Trans. Electron. (Japanese Edition), vol.J82-C-II, no.2, pp.56-68, Feb. 1999.

[2] T. Myono, A. Uemoto, S. Kawai, E. Nishibe, S. Kikuchi, T. Iijima, and H. Kobayashi, "High-efficiency charge-pump circuits with large current output for mobile equipment applications," IEICE Trans. Electron., vol.E84-C, no.10, pp.1602-1611, Oct. 2001.

[3] K. Min and J. Ahn, "CMOS charge pumps using cross-coupled charge transfer switches with improved voltage pumping gain and low gate-oxide stress for low-voltage memory circuits," IEICE Trans. Electron., vol.E85-C, no.1, pp.225-229, Jan. 2002.

[4] K. Yamada, N. Fujii, and S. Takagi, "Capacitance value free switched capacitor DC-DC voltage converter realizing arbitrary rational conversion ratio," IEICE Trans. Fundamentals, vol.E87-A, no.2, pp.344-349, Feb. 2004.

[5] K. Eguchi, F. Ueno, and T. Inoue, "Design of a 3/2 step-up SC DC-DC converter for diode-lamps," IEEJ Trans. EIS, vol.125, no.3, pp.528-529, 2005.

[6] H. Lee and P.K.T. Mok, "Switching noise and shoot-through current reduction techniques for switched-capacitor voltage doubler," IEEE J. Solid-State Circuits, vol.40, no.5, pp.1136-1146, 2005.

[7] B.R. Gregoire, "A compact switched-capacitor regulated charge pump power supply," IEEE J. Solid-State Circuits, vol.41, no.8, pp.1944-1953, 2006

[8] C.L. Wei, L.Y. Wu, H.H. Yang, C.H. Tsai, B.D. Liu, and S.J. Chang, "A versatile step-up/step-down switched-capacitor-based DC-DC converter," IEICE Trans. Electron., vol.E91-C, no.5, pp.809-812, May 2008

[9] T. Yamakawa, T. Inoue, and A. Tsuneda, "Design and experiments of a novel low-ripple Cockcroft-Walton AC-to-DC converter for a coil-coupled passive RFID tag," IEICE Trans. Fundamentals, vol.E91-A, no.2, pp.513-520, Feb. 2008.

[10] K. Eguchi, I. Oota, S. Terada, and T. Inoue, "A design method of switched-capacitor power converters by employing a ring-type power converter," Int. J. Innovative Computing, Information and Control, vol.5, no.10(A), pp.2927-2938, 2009. 